${ }^{1}$ Cardiología, Unidad de Medicina Cardiovascular Integrada, Hospital Las Higueras, Talcahuano, Chile.

${ }^{2}$ Cirugía Cardiaca, Unidad de Medicina Cardiovascular Integrada, Hospital Las Higueras, Talcahuano, Chile.

Trabajo no recibió financiamiento. Los autores declaran no tener conflicto de interés.

Recibido el 3 de diciembre de 2020, aceptado el 24 de febrero de 2021.

Correspondencia a: Dr. Osvaldo Pérez P. Unidad de Medicina

Cardiovascular Integrada, Hospital Las Higueras. Alto Horno \#777, Talcahuano, Chile. operpe@me.com

\section{Implante de válvula aórtica percutánea con técnica de sedación consciente}

\author{
RENÉ HAMEAU D. ${ }^{1}$, ALFONSO OLMOS C. ${ }^{1}$, CLAUDIA RODRÍGUEZ B. ${ }^{1}$, \\ GUSTAVO MERIÑO S. ${ }^{2}$, OSVALDO PÉREZ P. ${ }^{1}$

\section{Transfemoral transcatheter aortic valve implantation under conscious sedation. Experience in 15 patients}

Background: Transfemoral transcatheter aortic valve implantation (TAVI) is the standard of treatment for patients with symptomatic severe aortic stenosis (AE) and intermediate or high surgical risk. The use of conscious sedation (CS) could reduce complications and allow an early discharge of these patients. Aim: To report our experience with TAVI under conscious sedation. Material and Methods: Review of medical records of 15 patients aged $79 \pm 6$ years (53\% women) undergoing a transfemoral TAVI implant under conscious sedation. Results: The indications for the procedure were severe AE in 13 patients and biological prosthetic dysfunction in two. The mean Thoracic Surgeons predicted risk of mortality score was 7.3. The valves used were Edwards Sapien 3 in three patients, Medtronic Evolut in five, Boston Acurate Neo in four and Meril Myval in three. A successful implant was achieved in all cases and there were no hospital mortality or pacemaker requirements. One patient had a stroke, and one patient had a vascular access complication. Early discharge $(<72 \mathrm{~h})$ was achieved in $80 \%$ of patients. Conclusions: TAVI under conscious sedation was a safe procedure and associated with a complication rate similar to previous reports, allowing for an early hospital discharge in most patients.

(Rev Med Chile 2021; 149: 520-526)

Key words: Aortic Valve Stenosis; Conscious Sedation; Transcatheter Aortic Valve Replacement.

\footnotetext{
L
} os avances en el implante percutáneo de la válvula aórtica (TAVI) han transformado a la estenosis aórtica (EA) en la valvulopatía primaria más frecuentemente intervenida, ya sea quirúrgica o percutáneamente, tanto en Europa como en EEUU ${ }^{1}$.

Existe una búsqueda constante de simplificar esta técnica para optimizar el uso de recursos, disminuir las complicaciones y acortar la estadía hospitalaria. Esto ha motivado el desarrollo de técnicas llamadas "minimalistas" que incluyen el uso de sedación consciente (SC), modificaciones en la técnica de estimulación por marcapasos, desinvadir precozmente al paciente, etc ${ }^{2}$.
La adopción de estos protocolos parece ser una alternativa interesante en la realidad nacional, especialmente a nivel de establecimientos públicos, donde la búsqueda de estrategias costo-efectivas debe ser prioritario. El objetivo de este estudio es reportar los resultados de una serie de casos de implante de TAVI bajo SC en el Hospital Las Higueras, Talcahuano, Chile.

\section{Material y Métodos}

Estudio descriptivo de serie de casos sobre pacientes sometidos al implante de TAVI trans- 
femoral bajo sedación consciente en la Unidad de Medicina Cardiovascular Integrada del Hospital Las Higueras desde mayo de 2018 a junio de 2019. Los criterios para considerar el uso de SC fueron:

1) Hombres o mujeres y portadores de estenosis aórtica severa sintomática.

2) Acceso transfemoral adecuado.

3) Pacientes de alto riesgo quirúrgico o inoperables tras la discusión con el equipo de Cirugía Cardiaca (Heart team).

Se consideraron como criterios de exclusión para el uso de SC fueron:

1) Pacientes con valvulopatía aortica bicúspide.

2) Acceso femoral inadecuado.

3) Indicación de cirugía cardio-torácica por otro motivo (enfermedad coronaria, valvulopatía mitral o tricuspídea, patología aórtica, etc).

4) Shock cardiogénico.

El manejo de estos pacientes se realizó en 4 etapas:

\section{Evaluación e implante de TAVI}

Se estableció un policlínico de Estenosis Aórtica dedicado a la evaluación de los posibles candidatos a la terapia. Allí se realizaba la valoración clínica y se indicaba el estudio imagenológico correspondiente (Ecocardiograma TT (EcoTT), Angio-Tomografía computada (AngioTAC) y coronariografía en todos los pacientes). La indicación de TAVI se discutió caso a caso en "Heart Team” que incluyó a Cardiocirujanos y Cardiólogos intervencionistas.
El implante de TAVI se realizó en el Laboratorio de Hemodinamia con apoyo de EcoTT. Se empleó anestesia local y sedación consciente con Fentanilo \pm Midazolam para lograr una ansiolisis suave que permitiera el contacto verbal con el operador.

$\mathrm{El}$ acceso femoral fue pre-cerrado con 2 dispositivos Proglide Perclose (Abbott Vascular) + acceso radial izquierdo contralateral para inserción del pigtail en aquellos de menor riesgo de complicaciones. El acceso femoral contralateral se prefirió en aquellos con enfermedad vascular periférica significativa y con riesgo elevado de lesión vascular (disección, perforación, etc) u oclusión aguda.

Se instaló una sonda marcapasos transitoria (SMPT) vía venosa central en aquellos pacientes con alto riesgo de BAV o aquellos que recibirían una válvula Sapien 3, Myval y Evolut. En los demás casos, se realizó estimulación a través de la guía en el Ventrículo izquierdo (VI) según lo descrito en la literatura ${ }^{3}$.

Se utilizaron válvulas de tipo autoexpandibles: Evolut R (Medtronic, Minnesota, USA) o Acurate Neo (Boston Scientific, Massachusetts, USA) y balón-expandibles: Sapien 3 (EdwardsLifesciences, California, USA) o Myval (Meril, Gujarat, India) y se implantaron siguiendo las recomendaciones del fabricante. Los detalles de cada válvula se describen en la Tabla 1.

Se consideró un implante exitoso aquel que cumpliera los criterios VARC2 $2^{4}$ : Ausencia de mortalidad + adecuada posición anatómica del implante + correcto funcionamiento (sin mismatch

Tabla 1. Características de las prótesis valvulares aórticas utilizadas

\begin{tabular}{|c|c|c|c|c|c|c|c|}
\hline \multirow{2}{*}{$\begin{array}{l}\text { Tipo de } \\
\text { válvula }\end{array}$} & \multirow[t]{2}{*}{ Nombre } & \multicolumn{2}{|c|}{ Material } & \multirow{2}{*}{$\begin{array}{c}\text { Tamaño válvula } \\
\text { (mm) }\end{array}$} & \multirow{2}{*}{$\begin{array}{l}\text { Tamaño } \\
\text { introductor }\end{array}$} & \multirow[t]{2}{*}{ Posición } & \multirow[t]{2}{*}{ Reposicionable } \\
\hline & & Frame & Velos & & & & \\
\hline \multirow[t]{2}{*}{$\begin{array}{l}\text { Auto- } \\
\text { expandible }\end{array}$} & Evolut R & Nitinol & $\begin{array}{l}\text { Pericardio } \\
\text { porcino }\end{array}$ & $23,26,29$ у 34 & $\begin{array}{c}\text { 14F }(23,26 \\
29 \mathrm{~mm}) \\
16 \mathrm{~F}(34 \mathrm{~mm})\end{array}$ & $\begin{array}{l}\text { Supra- } \\
\text { anular }\end{array}$ & Sí \\
\hline & $\begin{array}{l}\text { Acurate } \\
\text { Neo }\end{array}$ & Nitinol & $\begin{array}{l}\text { Pericardio } \\
\text { porcino }\end{array}$ & 23,25 y 27 & $14 \mathrm{~F}$ & $\begin{array}{l}\text { Supra- } \\
\text { anular }\end{array}$ & No \\
\hline \multirow[t]{2}{*}{$\begin{array}{c}\text { Balón } \\
\text { expandible }\end{array}$} & Sapien 3 & $\begin{array}{l}\text { Cromo- } \\
\text { cobalto }\end{array}$ & $\begin{array}{l}\text { Pericardio } \\
\text { bovino }\end{array}$ & $20,23,26$ y 29 & $\begin{array}{c}14 \mathrm{~F}(20,23 \\
26 \mathrm{~mm}) \\
16 \mathrm{~F}(29 \mathrm{~mm})\end{array}$ & $\begin{array}{l}\text { Intra- } \\
\text { anular }\end{array}$ & No \\
\hline & Myval & $\begin{array}{l}\text { Cromo- } \\
\text { cobalto }\end{array}$ & $\begin{array}{l}\text { Pericardio } \\
\text { bovino }\end{array}$ & $\begin{array}{c}20,21,5,23 \\
24,5,26,27,5 \\
29,30,5 \text { у } 32\end{array}$ & $22 \mathrm{~F}$ & $\begin{array}{l}\text { Intra- } \\
\text { anular }\end{array}$ & No \\
\hline
\end{tabular}


prótesis-paciente, gradiente medio $<20 \mathrm{mmHg}$ y ausencia de leak moderado a severo).

El equipo a cargo del procedimiento consistió en: Dos Cardiólogos Intervencionistas para el implante, un Cardiólogo para apoyo con ETT y monitoreo hemodinámico, una Enfermera de pabellón para administración de medicamentos + monitoreo y una Técnico paramédico.

\section{Postoperatorio inmediato}

Se retiraron todos los accesos vasculares centrales en pabellón utilizando los dispositivos de cierre. La SMPT se retiró en pabellón si el paciente no presentaba de trastornos del ritmo significativos (BCRI, Bloqueo AV de alto grado, Bradicardia extrema) o, en ausencia de complicaciones, dentro de las primeras $24 \mathrm{~h}$ siguiendo lo descrito en la literatura ${ }^{5}$. Todos los pacientes fueron trasladados a la Unidad Coronaria para monitorización.

\section{Control pre-alta}

Se realizaba un EcoTT, ECG y exámenes de laboratorio. De acuerdo a la evolución clínica (incluyendo deambulación precoz), se indicaba el alta y se agendaba el control ambulatorio. Se definió un alta precoz como aquella ocurrida $\leq 72 \mathrm{~h}$ desde el implante.

\section{Control ambulatorio}

Seguimiento en Policlínico de Cardiología a los 30 días.

El objetivo de este reporte fue valorar la seguridad del protocolo, evaluando las complicaciones intraprocedimiento e intrahospitalarias (vasculares, tasa de marcapsos definitivo, leak paravalvular) y evolución clínica (días de hospitalización, mortalidad a 30 días, etc). También buscaremos describir el perfil de pacientes intervenidos así como los detalles técnicos de cada intervención (Ej: duración del procedimiento, tipo de sedación utilizada y sus dosis, etc).

El diseño de este estudio y su contenido fueron aprobados íntegramente por el Comité de Ética correspondiente.

\section{Análisis de datos}

Mediante la revisión de los registros clínicos se caracterizaron las variables del procedimiento, eventos adversos, parámetros hemodinámicos así como ecocardiográficos, antecedentes clínicos y de laboratorio de cada sujeto. Para la descripción de variables cualitativas se empleó tablas de frecuencia. Las variables cuantitativas se describieron con la media \pm desviación estándar si su distribución es normal. En caso contrario, se utilizó mediana + valores min-máx. Cálculos estadísticos realizados con STATA 14.

\section{Resultados}

En el período entre mayo de 2018 y junio de 2019, un total de 15 pacientes fueron sometidos TAVI bajo SC. El 53,3\% eran de sexo femenino y la edad promedio fue de $79 \pm 6,5$ años.

Las comorbilidades más frecuentes fueron la Hipertensión arterial (80\%) e historia de insuficiencia cardiaca $(86 \%)$. La mitad de los pacientes era fumador, cerca del $60 \%$ tenía antecedentes de Infarto agudo al miocardio (IAM) previo y 46\% de Diabetes Mellitus tipo 2. El 46\% refería historia previa de angioplastía coronaria y $40 \%$ de cirugía de revascularización (Tabla 2). Un paciente presentaba el antecedente de obesidad mórbida.

La fracción de eyección del ventrículo izquierdo (FEVI) promedio fue de $51 \pm 8,7 \mathrm{mmHg}$ y el gradiente aórtico medio basal fue 56,8 $\pm 12,0$ mmHg. La mortalidad a 30 días por score STSPROM fue de $7,32 \%$, con un mínimo de 3 y un máximo de 12,4 puntos. La decisión de TAVI en el paciente de bajo riesgo estuvo dada por el antecedente de cirugía de revascularización previa por lo que la reoperación fue descartada por el equipo quirúrgico. Las principales indicaciones de TAVI fueron EA severa sintomática $(\mathrm{n}=12)$ y disfunción de prótesis biológica $(\mathrm{n}=3)$.

La vía femoral derecha fue la más utilizada $(80 \%)$ y se asoció en su mayoría a un 2 do acceso radial contralateral para inserción de un pigtail 5F. Se contó con apoyo de Ecocardiograma TT intra-pabellón.

La sedación consciente se logró principalmente con el uso de Fentanilo (promedio $93 \pm 34 \mathrm{ug}$ ); en ocasiones, asociado a Midazolam en dosis bajas. La duración promedio del implante fue 79,1 \pm 43,2 min y la dosis promedio de contraste utilizada fue $156 \mathrm{ml}$.

Se utilizó un marcapasos transitorio por vía venosa central en 11 pacientes y en 4 casos se estimuló directamente a través de la guía de alto soporte en el ventrículo izquierdo. 
Tabla 2. Características clínicas de los pacientes

\begin{tabular}{|c|c|c|c|c|c|c|c|c|c|c|c|c|c|}
\hline $\begin{array}{c}\text { Pa- } \\
\text { ciente }\end{array}$ & Edad & Sexo & HTA & DM & DLP & Tabaco & $\begin{array}{c}\text { IAM } \\
\text { antiguo }\end{array}$ & $\begin{array}{l}\text { ERC en } \\
\text { hemo- } \\
\text { diálisis }\end{array}$ & $\begin{array}{c}\text { PCI } \\
\text { previa }\end{array}$ & $\begin{array}{c}\text { CRM } \\
\text { previa }\end{array}$ & $\begin{array}{l}\text { FEVI } \\
(\%)\end{array}$ & $\begin{array}{c}\text { Gradiente } \\
\text { aórtico } \\
\text { medio } \\
(\mathbf{m m H g})\end{array}$ & $\begin{array}{c}\text { STS } \\
\text { score }\end{array}$ \\
\hline 1 & 64 & $\mathrm{H}$ & + & + & - & - & + & - & - & + & 35 & 61 & 5,2 \\
\hline 2 & 67 & $\mathrm{H}$ & + & - & - & + & + & - & + & + & 35 & 43 & 3,0 \\
\hline 3 & 81 & $\mathrm{M}$ & - & - & + & + & - & - & - & - & 60 & 60 & 8,3 \\
\hline 4 & 88 & M & - & - & - & + & - & - & & - & 55 & 52 & 6,4 \\
\hline 5 & 81 & $\mathrm{H}$ & + & + & - & - & + & - & + & + & 42 & 50 & 8,3 \\
\hline 6 & 79 & $\mathrm{H}$ & + & + & - & + & + & - & - & + & 45 & 53 & 7,7 \\
\hline 7 & 82 & $\mathrm{H}$ & + & + & + & - & + & - & + & - & 48 & 50 & 4,0 \\
\hline 8 & 84 & M & - & - & - & + & - & - & - & - & 60 & 71 & 10,0 \\
\hline 9 & 84 & M & + & + & - & + & - & - & - & - & 60 & 80 & 7,6 \\
\hline 10 & 79 & M & + & - & - & + & - & - & - & - & 50 & 61 & 11,7 \\
\hline 11 & 74 & M & + & - & - & + & + & - & + & - & 50 & 70 & 4,9 \\
\hline 12 & 85 & M & + & - & + & - & - & - & - & - & 60 & 43 & 12,4 \\
\hline 13 & 79 & $M$ & + & + & + & - & + & - & + & - & 55 & 60 & 8,3 \\
\hline 14 & 84 & $\mathrm{H}$ & + & + & - & - & + & + & + & + & 50 & 71 & 6,0 \\
\hline 15 & 80 & $\mathrm{H}$ & + & - & + & - & + & - & + & + & 60 & 40 & 6,0 \\
\hline
\end{tabular}

H: Hombre, M: Mujer, HTA: Hipertensión arterial, DM: Diabetes Mellitus, DLP: Dislipidemia, IAM: Infarto agudo al miocardio, ERC: Enfermedad renal crónica, PCl: Angioplastía coronaria, CRM: Cirugía de revascularización miocárdica, FEVI: Fracción de eyección del ventrículo izquierdo, (+): Factor de riesgo presente, (-): Factor de riesgo ausente.

Los tipos de prótesis utilizadas fueron: Edwards SAPIEN 3 ( $\mathrm{n}=3$ ); Medtronic Evolut $(\mathrm{n}=5)$; Boston Acurate Neo $(\mathrm{n}=4)$ y Meril Myval $(\mathrm{n}=3)$. El $20 \%$ correspondió a TAVI valve-in-valve. No hubo necesidad de conversión a anestesia general en ningún caso y solo en $2(13,3 \%)$ pacientes se requirió postdilatar. Los criterios de éxito del implante se lograron en todos los pacientes, con un gradiente valvular medio post procedimiento de $5,4 \pm 3,9 \mathrm{mmHg}$ y ausencia de leak paravalvular moderado-severo en todos ellos.

La media de hospitalización fue de 2,8 \pm 1,7 días (Rango 1- 6 días) y el alta precoz se logró en el $80 \%$ de ellos.

Respecto de las complicaciones, 1 paciente con obesidad mórbida requirió cierre vascular quirúrgico por falla del dispositivo percutáneo y 1 paciente presentó un accidente vascular (ACV) cerebeloso con recuperación completa a los 2 meses. No hubo muertes, requerimientos de marcapasos ni falla renal aguda con necesidad de hemodiálisis a 30 días (Tabla 3). La totalidad de los pacientes fue dado de alta bajo terapia antiplaquetaria dual.

\section{Discusión}

Descrita por primera vez en el año $2008^{6}$, la técnica de implante de TAVI bajo SC ha sido adoptada de forma creciente por numerosos centros y sus resultados han sido comparables al uso de la anestesia general ${ }^{7}$. Esto explicaría que solo 17\% de los hospitales en EEUU siguen utilizando exclusivamente la anestesia general en sus procedimientos.

La mayor parte de la evidencia actual proviene de registros clínicos: Eskandari et al. ${ }^{8}$ reportó la experiencia de 2.243 TAVI implantadas con o sin anestesia general. La estrategia menos invasiva mostró tasas similares de mortalidad a 1 año, éxito del implante o insuficiencia aórtica residual. El estudio Vancouver $3 \mathrm{M}^{9}$ mostró los resultados de 411 pacientes en 13 centros de Canadá y EEUU con esta técnica. La mortalidad a 30 días fue de $2,9 \%$, logrando el alta al día siguiente en $80 \%$ de los pacientes y dentro de $48 \mathrm{~h}$ en casi $90 \%$. El implante de marcapasos definitivo ocurrió en 5,7\% y las complicaciones vasculares en $2,4 \%$. Estos 
Tabla 3. Descripción del procedimiento y resultados inmediatos

\begin{tabular}{|c|c|c|c|c|c|c|c|c|c|c|c|}
\hline $\begin{array}{c}\text { Pa- } \\
\text { ciente }\end{array}$ & $\begin{array}{c}\text { Dosis } \\
\text { fenta- } \\
\text { nilo } \\
\text { (ug) }\end{array}$ & $\begin{array}{c}\text { Dosis } \\
\text { mida- } \\
\text { zolam } \\
\text { (mg) }\end{array}$ & $\begin{array}{l}\text { Tipo de } \\
\text { válvula }\end{array}$ & $\begin{array}{c}\text { Valve } \\
\text { in } \\
\text { valve }\end{array}$ & $\begin{array}{c}\text { Duración } \\
\text { procedi- } \\
\text { miento } \\
\text { (min) }\end{array}$ & $\begin{array}{c}\text { Duración } \\
\text { estadía } \\
\text { hospita- } \\
\text { laria } \\
\text { (días) }\end{array}$ & $\begin{array}{c}\text { Compli- } \\
\text { caciones } \\
\text { vascu- } \\
\text { lares } \\
\text { mayores }\end{array}$ & $\begin{array}{c}\text { Implante } \\
\text { de mar- } \\
\text { capasos } \\
\text { definiti- } \\
\text { vo }\end{array}$ & $\begin{array}{l}\text { Morta- } \\
\text { lidad } \\
\text { IH }\end{array}$ & $\begin{array}{l}\text { ACV } \\
\text { a } 30 \\
\text { días }\end{array}$ & $\begin{array}{c}\text { Leak } \\
\text { mode- } \\
\text { rado o } \\
\text { severo }\end{array}$ \\
\hline 1 & 100 & 0 & Myval \#23 & Sí & 85 & 2 & 0 & 0 & 0 & 0 & 0 \\
\hline 2 & 100 & 5 & Myval \#26 & No & 105 & 1 & 0 & 0 & 0 & 0 & 0 \\
\hline 3 & 75 & 1 & Myval \#26 & No & 190 & 6 & 0 & 0 & 0 & 0 & 0 \\
\hline 4 & 50 & 0 & $\begin{array}{c}\text { Acurate Neo } \\
\# 24\end{array}$ & No & 96 & 1 & 1 & 0 & 0 & 0 & 0 \\
\hline 5 & 50 & 0 & $\begin{array}{c}\text { Acurate Neo } \\
\quad \# 27\end{array}$ & No & 87 & 6 & 0 & 0 & 0 & 1 & 0 \\
\hline 6 & 100 & 0 & $\begin{array}{c}\text { Acurate Neo } \\
\# 27\end{array}$ & Sí & 68 & 2 & 0 & 0 & 0 & 0 & 0 \\
\hline 7 & 100 & 0 & $\begin{array}{c}\text { Acurate Neo } \\
\quad \# 27\end{array}$ & No & 100 & 2 & 0 & 0 & 0 & 0 & 0 \\
\hline 8 & 50 & 0 & Sapien 3 \#26 & No & 78 & 1 & 0 & 0 & 0 & 0 & 0 \\
\hline 9 & 75 & 0 & Sapien 3 \#26 & No & 62 & 6 & 0 & 0 & 0 & 0 & 0 \\
\hline 10 & 150 & 0 & Evolut R \#26 & No & 60 & 2 & 0 & 0 & 0 & 0 & 0 \\
\hline 11 & 100 & 0 & Evolut R \#29 & No & 113 & 2 & 0 & 0 & 0 & 0 & 0 \\
\hline 12 & 75 & 0 & Evolut R \#26 & No & 125 & 3 & 0 & 0 & 0 & 0 & 0 \\
\hline 13 & 75 & 0 & Evolut R \#26 & No & 180 & 3 & 0 & 0 & 0 & 0 & 0 \\
\hline 14 & 150 & 0 & Sapien 3\#26 & Sí & 180 & 2 & 0 & 0 & 0 & 0 & 0 \\
\hline 15 & 150 & 0 & Sapien 3 \#26 & No & 68 & 3 & 0 & 0 & 0 & 0 & 0 \\
\hline
\end{tabular}

$\mathrm{IH}$ : Intrahospitalaria, ACV: Accidente cerebrovascular isquémico.

resultados fueron similares tanto en centros de alto, mediano o bajo volumen.

Otra experiencia reciente es aquella reportada por Butala et al. ${ }^{11}$, quien analizó un registro de $>120.000$ pacientes del registro TVT entre 2016 y 2019. Destaca un incremento en el uso de SC desde $33 \%$ a $64 \%$. En este estudio, el uso de SC se asoció a una modesta pero significativa disminución en la mortalidad intrahospitalaria (diferencia de riesgo ajustado: $0,2 \%, p 0,01$ ), mortalidad a 30 días (diferencia de riesgo ajustado: 0,5\%, $\mathrm{p}<0,001$ ) y estadía hospitalaria (diferencia de riesgo ajustado: $0,8$ días, $\mathrm{p}<0,001)$.

Ichibori ${ }^{10}$ incluso reporta el uso de SC en TAVI de urgencia $(n=77)$, definidas como aquellos sujetos que recibieron la válvula en la misma hospitalización en que ingresaron por paro cardiaco, falla cardiaca severamente descompensada, síndrome coronario agudo o síncopes a repetición vs TAVI en pacientes electivos $(\mathrm{n}=392)$. No hubo diferencias respecto del IAM, complicaciones vasculares, ACV o mortalidad intrahospitalaria a 30 días vs. la anestesia general.

El estudio SOLVE TAVI ${ }^{12}$ es el ensayo multicéntrico más recientemente publicado y utilizó un diseño 2 × 2 para comparar anestesia general vs sedación consciente y una válvula autoexpandible vs una balón-expandible. Este aleatorizó a 447 pacientes a TAVI transfemoral con SC vs anestesia general y no encontró diferencias en el desenlace compuesto de mortalidad por cualquier causa, ACV, infarto agudo al miocardio (IAM), infección con requerimientos de antibióticos o falla renal aguda a 30 días ( $27 \%$ vs $26,4 \%$, p 0,02 para equivalencia). Tampoco hubo diferencias significativas al analizar mortalidad (3,2\% vs $2,3 \%, \mathrm{p}<0,001$ para equivalencia), infarto agudo al miocardio, ACV o falla renal aguda. Hubo un menor requerimiento 
de inótropos y vasopresores en el grupo de SC $(62,8 \% \text { vs } 97,3 \% \text {, IC95\%; - } 41 \text { a }-27)^{13}$.

Los cuestionamientos a esta estrategia son diversos: En primer lugar, está la carencia del apoyo con Ecocardiograma Transesofágico, recordando que algunas series lo asocian a una reducción de eventos clínicos a largo plazo ${ }^{14}$. También se plantea que estos reportes pudieran tener sesgos de selección de pacientes, asignando la estrategia minimalista a la población menos enferma o respecto de la terapia recibida, considerando que aquellos operadores con mayor volumen estarán más abiertos a probar esta técnica. Otros autores abogan por el apoyo siempre de un Anestesiólogo intrapabellón: Mayr et al. ${ }^{15}$ reporta $10 \%$ de eventos adversos bajo SC en un centro de alto volumen y donde el más frecuente fue la conversión a anestesia general.

También existen aprensiones respecto de desinvadir precozmente a los pacientes, sin embargo, conductas como el retiro precoz de la SMPT (incluso antes de salir de pabellón) se encuentran validadas por la literatura y deben valorarse caso a caso en base a la presencia de predictores o aparición de trastornos eléctricos intra procedimiento ${ }^{5}$. Finalmente, Pineda et cols ${ }^{16}$ reportó una tasa de complicaciones que requirieron cirugía de rescate solo de $1,17 \%$ al analizar $>47.500$ pacientes sometidos a TAVI por lo que la presencia del equipo Cardioquirúrgico en el pabellón debe ser evaluada caso a caso según el perfil de riesgo.

Esta serie incluyó a 15 pacientes con implante de TAVI con un bajo SC; la mayoría de riesgo quirúrgico intermedio y con una edad promedio de 79 años; similar a la reportada por otros estudios recientes $^{17,18}$.

Las fortalezas de este reporte se basan en que la estrategia de SC fue aplicada de forma sistemática en todos los pacientes, utilizando prótesis tanto balón-expandibles como auto-expandibles y sobre válvulas nativas como valve-in-valve. Por otro lado, dado que este centro fue uno de los primeros a nivel nacional en utilizar la SC; la presente constituye la serie más grande publicada en el país.

También reportamos el uso exitoso de técnicas novedosas como la estimulación de marcapasos directamente a través de la guía en el VI. En nuestra experiencia, se trata de técnica segura que no compromete el éxito del implante y evita la necesidad de instalar un acceso venoso central adicional. Experiencias internacionales sugieren que esta estrategia pudiera reducir la duración del implante, el tiempo de fluoroscopía y los costos del procedimiento ${ }^{19}$.

En conjunto, estas intervenciones permiten una reducción marcada del tiempo operatorio respecto de aquellas realizados bajo anestesia general. En esta serie, el promedio global fue 79 min (72 min si se excluye la paciente que presentó una complicación vascular); duración inferior a lo reportado por otras series nacionales ${ }^{20} \mathrm{y}$ muy cercana a lo descrito en centros extranjeros de alto volumen'.

Finalmente, el alta precoz puede ser una ventaja considerando la sobrecarga al sistema de salud generada por la actual pandemia del COVID-19, permitiendo mantener una baja tasa de ocupación de camas.

Respecto de las limitaciones, debemos mencionar que se trata de un reporte unicéntrico por lo que sus resultados pudieran no ser reproducibles en otros hospitales. Además, se trata de un volumen de pacientes bajo en comparación con otras series internacionales y tampoco existe internacionalmente un protocolo estándar de sedación consciente por lo que está práctica presenta una variabilidad inherente dependiendo del centro donde se realice.

En cuanto a la experiencia nacional, Maluenda et al. ya ha reportado la costo-efectividad de TAVI vs el manejo conservador ${ }^{21}$. El primer reporte en Chile del uso de SC corresponde a una serie de 10 pacientes por Hameau et al. ${ }^{20}$. En esa publicación se describe un adecuado perfil de seguridad asociado a estadías hospitalarias reducidas (mediana de hospitalización: 2 días), si bien se trata exclusivamente de prótesis auto-expandibles.

En conclusión, este trabajo corresponde a la mayor serie nacional publicada con el uso de sedación consciente para el implante de TAVI y sugiere que se trata de un procedimiento seguro en nuestro medio. La creación de protocolos locales y la adecuada selección de pacientes son claves para lograr resultados satisfactorios con esta técnica.

\section{Referencias}

1. Nkomo VT, Gardin JM, Skelton TN, Gottdiener JS, Scott CG, Enriquez-Sarano M. Burden of valvular heart diseases: a population-based study. Lancet. 2006; 368 (9540): 1005-11. 
2. Lester L, Brady MB, Brown CH. Sedation Versus General Anesthesia for TAVR: Where Do We Go From Here? J Cardiothorac Vasc Anesth. 2017; 31 (6): 2055-7.

3. Faurie B, Abdellaoui M, Wautot F, Staat P, Champagnac $\mathrm{D}$, Wintzer-Wehekind J, et al. Rapid pacing using the left ventricular guidewire: Reviving an old technique to simplify BAV and TAVI procedures. Catheter Cardiovasc Interv. 2016; 88 (6): 988-93.

4. Pieter Kappetein A, Head S, Généreux P, Piazza N, Van Mieghem N, Blackstone E, et al. Updated standardized endpoint definitions for transcatheter aortic valve implantation: The Valve Academic Research Consortium-2 consensus document. Eur Heart J. 2012; 33 (1): 2403-18.

5. Auffret V, Puri R, Urena M, Chamandi C, Rodriguez-Gabella T, Philippon F, et al. Conduction disturbances after transcatheter aortic valve replacement: Current status and future perspectives. Circulation. 2017; 136: 1049-69.

6. Behan M, Haworth P, Hutchinson N, Trivedi U, Laborde JC, Hildick-Smith D. Percutaneous aortic valve implants under sedation: Our initial experience. Catheter Cardiovasc Interv. 2008; 72 (7): 1012-5.

7. Villablanca P, Mohananey D, Nikolic K, Bangalore S, Slovut D, Mathew V, et al. Comparison of local versus general anesthesia in patients undergoing transcatheter aortic valve replacement: A meta-analysis. Catheter Cardiovasc Interv. 2018; 91 (2): 330-42.

8. Eskandari M, Aldalati O, Dworakowski R, Byrne J, Alcock E, Wendler O, et al. Comparison of general anaesthesia and non-general anaesthesia approach in transfemoral transcatheter aortic valve implantation. Heart. 2018; 104 (19): 1621-8.

9. Wood D, Lauck S, Cairns JHK, Cook R, Welsh R, Leipsic J, et al. The Vancouver 3M (Multidisciplinary, Multimodality, But Minimalist) Clinical Pathway Facilitates Safe Next-Day Discharge Home at Low-, Medium-, and High-Volume Transfemoral Transcatheter Aortic Valve Replacement Centers: The 3M TAVR Study. JACC Cardiovasc Interv. 2019; 12 (5): 459-69.

10. Ichibori Y, Li J, Patel T, Lipinski J, Ladas T, Saric P, et al. Short-Term and Long-Term Outcomes of Patients Undergoing Urgent Transcatheter Aortic Valve Replacement Under a Minimalist Strategy. J Invasive Cardiol. 2019; 31 (2): 30-6.

11. Butala N, Chung M, Secemsky E, Manandhar P, Marquis-Gravel G, Kosinski A, et al. Conscious Sedation Versus General Anesthesia for Transcatheter Aortic Valve Replacement: Variation in Practice and Outcomes.
JACC Cardiovasc Interv. 2020; 13 (11): 1277-87.

12. Thiele H, Kurz T, Feistritzer H, Stachel G, Hartung P, Eitel I, et al. Comparison of newer generation self-expandable vs. balloon-expandable valves in transcatheter aortic valve implantation: the randomized SOLVE-TAVI trial. Eur Heart J. 2020; 41: 1890-9.

13. Thiele H, Kurz T, Feistritzer H, Stachel G, Hartung P, Lurz P, et al. General Versus Local Anesthesia with Conscious Sedation in Transcatheter Aortic Valve Implantation: The Randomized SOLVE-TAVI Trial. Circulation. 2020; 142 (1): 1437-47.

14. De Brito F, Carvalho L, Sarmento-Leite R, Mangione J, Lemos P, Siciliano A, et al. Outcomes and predictors of mortality after transcatheter aortic valve implantation: Results of the Brazilian registry. Catheter Cardiovasc Interv. 2015; 85 (5): E153-62.

15. Mayr P, Wiesner G, Husser O, Joner M, Michel J, Knorr J, et al. Critical adverse events during transfemoral TAVR in conscious sedation. Is an anesthesiologic support mandatory? Cardiovasc Revascularization Med. 2018; 19 (6): 41-6.

16. A. Pineda, J. Harrison, N. Kleiman, C. Rihal NB. Incidence and outcomes of surgical bailout during TAVR. JACC Cardiovasc Interv. 2019; 12 (18): 1751-64.

17. Thyregod H, Steinbrüchel D, Ihlemann N, Nissen H, Kjeldsen B, Petursson P, et al. Transcatheter versus surgical aortic valve replacement in patients with severe aortic valve stenosis: 1-year results from the all-comers NOTION randomized clinical trial. J Am Coll Cardiol. 2015; 65 (20): 2184-94.

18. León M, Smith C, Mack M, Makkar R, Svensson L, Kodali S, et al. Transcatheter or Surgical Aortic-Valve Replacement in Intermediate-Risk Patients. N Engl J Med. 2016; 374: 1609-20.

19. Faurie B, Souteyrand G, Staat P, Godin M, Caussin C, Van Belle E, et al. Left Ventricular Rapid Pacing Via the Valve Delivery Guidewire in Transcatheter Aortic Valve Replacement. JACC Cardiovasc Interv. 2019; 12 (24): 2449-59.

20. Hameau R, Veas N, Winter J, Valdebenito M, Muñoz R, Fuensalida A, et al. Enfoque minimalista en el implante de válvula aórtica percutánea. Rev Chil Cardiol. 2019; 38 (3): 173-81.

21. Maluenda G, Sepúlveda E, Alfaro M, Arias A, Muñoz R, León L, et al. Costo-Efectividad de Reemplazo Valvular Aórtico Percutáneo vs Terapia Conservadora en la Estenosis Aórtica de muy alto riesgo en un centro terciario chileno. Rev Chil Cardiol. 2015; 15 (1): 11-7. 